\title{
Concluding remarks: The importance of polymer science for biological systems
}

\section{David Tirrell*}

\author{
Received 22nd May 2008, Accepted 10th June 2008 \\ First published as an Advance Article on the web 23rd July 2008 \\ DOI: $10.1039 / \mathrm{b808690g}$
}

\begin{abstract}
The 139th Faraday Discussion covered many interesting subjects, divided into four sections: Cell Interactions; Membranes \& Walls; Proteins and Polysaccharides; and Natural \& Synthetic Polymers. Here, the meeting is summarised and future prospects are explored.
\end{abstract}

On behalf of all of the conference participants, I'd like to begin by thanking Tony Ryan and Athene Donald, and the rest of the Scientific Committee, for organizing an outstanding Faraday Discussion. The RSC staff has also done an excellent job on behalf of all of us.

I'd like to offer some impressions of what I've heard over the last three days. The title of the Discussion, The Importance of Polymer Science for Biological Systems, suggests that ideas, methods and perspectives drawn from polymer science should help us understand how biological systems behave. I believe that this assumption is true, and that we've seen evidence for it in the papers presented here.

What does the polymer science community have to offer in this regard? First and foremost, perhaps, is a strong grounding in macromolecular structure and in the processes of structure formation (crystallization, self-assembly, aggregation) in macromolecular systems. The connections between structure and physical properties (e.g., elasticity, viscous and viscoelastic behavior, and adhesion) have also been explored in substantial depth by polymer physicists and chemists. By my count, 17 of the 24 papers discussed at the conference addressed issues of this kind. We have experimental, theoretical and computational tools that are well suited to the study of biological systems. We have quantitative skills that are rare (though less rare than before) in the biological sciences, and synthetic skills that allow us to probe and engineer biology in new ways. And we bring new and useful (though often naïve) perspectives on biological behavior.

What else do we need? Most important, I believe, is a deeper appreciation of biological control mechanisms, including gene expression and cellular signaling pathways. Cells, tissues, and organisms respond to their environments through chemical as well as physical changes, and if we are to use polymer science to probe (or ultimately, to engineer) biological systems, we must understand the molecular mechanisms by which such changes occur. We must master the tools that are used to examine biological processes, to monitor gene expression, protein synthesis, protein turnover and post-translational modification. Such tools are in fact quite accessible to physical scientists who make the effort to acquire them. And we must listen carefully to our colleagues in biology in order to understand what the compelling biological questions really are. We needn't turn ourselves into biologists, but we must make an effort to communicate with our colleagues on their terms and in greater depth.

Division of Chemistry and Chemical Engineering, California Institute of Technology, 1200 East California Blvd, Pasadena, California, 91125,USA. E-mail: tirrell@caltech.edu 
My own laboratory has for many years been developing approaches to macromolecular science that blur the lines between polymer science and biology. By using artificial genes to direct the synthesis of artificial proteins in bacterial cells, we've been able to create new macromolecules characterized by complete control of chain length, sequence, and stereochemistry. We've introduced dozens of new amino acids into the proteins of bacterial and mammalian cells, and we've demonstrated the utility of new amino acids in biomaterials design, protein modification, protein evolution, and proteomic analysis. Much of this work has been done in collaboration with colleagues in the biological sciences: colleagues who have helped us acquire the needed skills and tools and pointed us toward compelling biological questions. It's been a rewarding endeavor, and I look forward to increasingly important ties between polymer science and the biological sciences in the future. 